\title{
Synthesis and Antimicrobial Evaluation of Some New Heterocyclic Compounds From Thienopyridines and Thienopyrimidine Derivatives
}

\author{
Abdullah A. Al-Karim Al-Shara'ey ${ }^{1}$, Eman Ali Thabet ${ }^{1}$, Fathia Mohammad Qasem Qaid ${ }^{1}$ and Faisal \\ M. Aqlan ${ }^{2,3}$ \\ ${ }^{I}$ Department of Chemistry, Faculty of Applied Science, Taiz University, Yemen. \\ ${ }^{2}$ Department of Chemistry, Faculty of Science, University of Jeddah, KAS. \\ ${ }^{3}$ Department of Chemistry, Faculty of Science, IBB University, Yemen. \\ E. mail: fethiyemohammed@gmail.com
}

\begin{abstract}
H)-thiones 1a,b were prepared and reacted with chloroacetonitrile to furnish 3-amino-4-aryl-2-carbamoyl-5-ethoxycarbonyl-6methyl thieno[2,3-b]pyridine derivative $3 \mathrm{a}, \mathrm{b}$. The reaction of $3 \mathrm{a}$ with a variety of reagent namely, ethylenediamine, sodium azide, tri ethylorthoformate, 4-chlorobenzaldehyde, carbondisulfide and sodium nitrous acid have been carried out and their products were identified. Most of these products were subjected to further reaction to obtain heterocyclic compounds. The all synthesized derivatives displayed good Antimicrobial activities against some species of bacteria as (E.colie, P.seudemones and S.aurous) and some species of fungal as (Aspergillus, Pencillium and Clado)
\end{abstract}

Keywords: Fused heterocyclic system; pyridothienopyrimidine, pyrazolo pyridothienopyrimidine, pyridothienoditriazine and triazolopyridothieno pyrimidine.

\section{1- Introduction:}

Thieno[2,3-b]pyridine ring system has proved to be an interesting class of heterocyclic (1). It has been reported that thienopyridine and pyridothieno pyrimidine derivatives extensively showed variety of activities such as , antibacterial (2-4), antimicrobial (5-6), diabetes mellitus (7-8), analgesic (9-10) and antipyretic (11). Thus, in continuation of our interest in the synthesis of heterocyclic compounds with expected biological activities (12-17), we report herein a convenient routes for the synthesis of novel heterocyclic compounds utilizing thieno pyridine and pyrazolopyridine derivatives.

\section{2- Result and Discussion:-}

$$
\begin{aligned}
& \text { the reaction of 4-aryl-3-cyano-5- } \\
& \text { ethoxycarbonyl-6-methylpyridine-2- }(1 H) \text { - } \\
& \text { thiones 1a,b with chloroacetonitrile in the } \\
& \text { presence of sodium acetate gave 2-substituted } \\
& \text { methylthio-4-aryl-3-cyano-5-ethoxycarbonyl-6- } \\
& \text { methyl pyridines 2a,b. Upon treatment of these } \\
& \text { compounds with sodium ethoxide in ethanol, } \\
& \text { they underwent intermolecular Thorpe-Ziegler } \\
& \text { cyclization to furnish 3-amino-4-aryl-2- } \\
& \text { carbamoyl-5-ethoxycarbonyl-6-methylthieno }
\end{aligned}
$$


[2,3-b] pyridine 3a,b. The latter thienopyridine derivatives were also prepared via direct reaction of compounds $1 \mathrm{a}, \mathrm{b}$ with chloro acetonitrile in the presence of excess of sodium ethoxide (17), (Scheme 1).

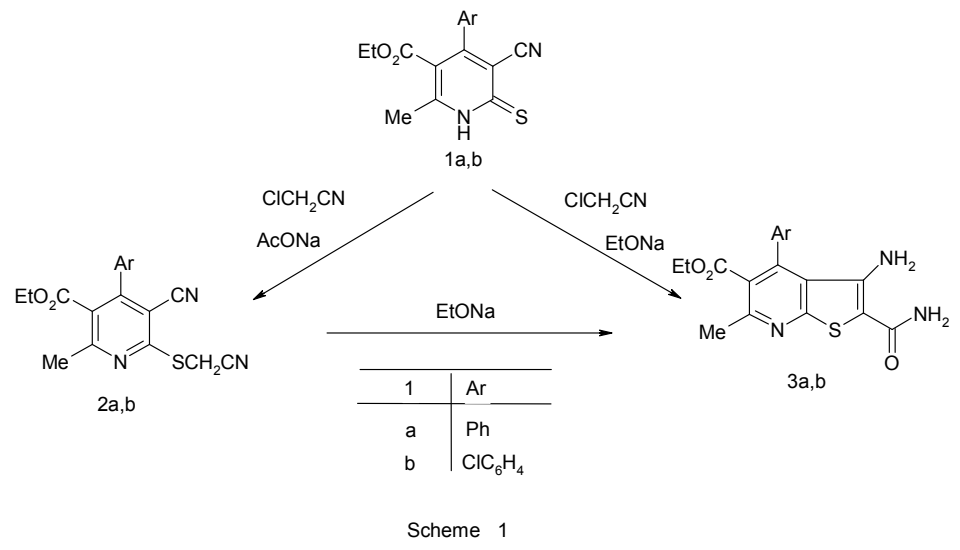

The reaction of 3a with ethylenediamine gave 3-amino-2-(4,5-dihydroimidazol-2-yl)-5ethoxycarbonyl-6-methyl-4-phenylthieno[2,3b]pyridine (4). Treatment of 3a with sodium azide in the presence of ammonium chloride resulted in the formation of the tetrazolyl compound 5. (Scheme 2).

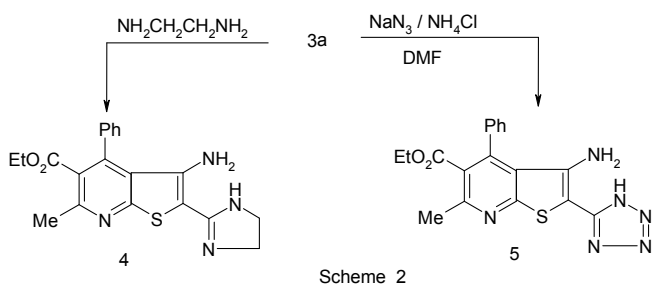

The reaction of 4 with triethylorthoformate, 4-chlorobenzaldehyde and carbondisulfide gave dihydroimidazolopyridothienopyrimidine derivatives 6,7 and 8 respectively. On treatment of 4 with sodium nitrate and sulfuric acid the dihydroimidazolopyridothienotriazine derivative 9 was obtained. (Scheme 3).

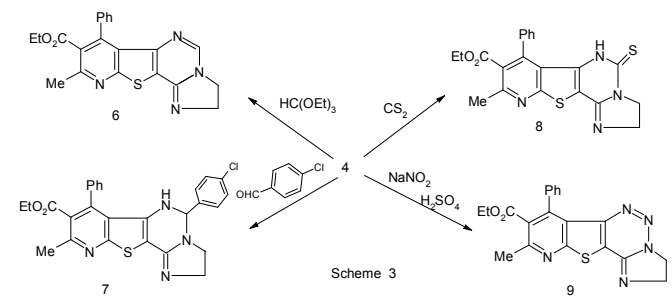

In the same manner, the compound 5 was reacted with triethylorthoformate under neat conditions and carbondisulfide in pyridine refluxing condition to produce the fused tetra cycliccompounds 10 and 11 respectively. (Scheme 4).

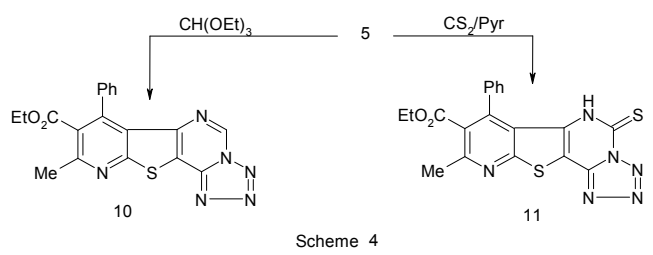

Diazotization of $3 \mathrm{a}$ using sodium nitrite in hydrochloric acid and acetic acid mixture gave 4-chloro-8-ethoxycarbonyl-7-methyl-9-phenyl pyrido[3`,2`:4,5]thieno[3,2-e][1,2,3]triazine (12). The reaction of 12 with hydrazine hydrate produced the hydrazinophenylpyrido thienotriazine derivative 13 , which was cyclocondensed with acetylacetone to furnish the pyridothienoditriazines 14. (Scheme 5).
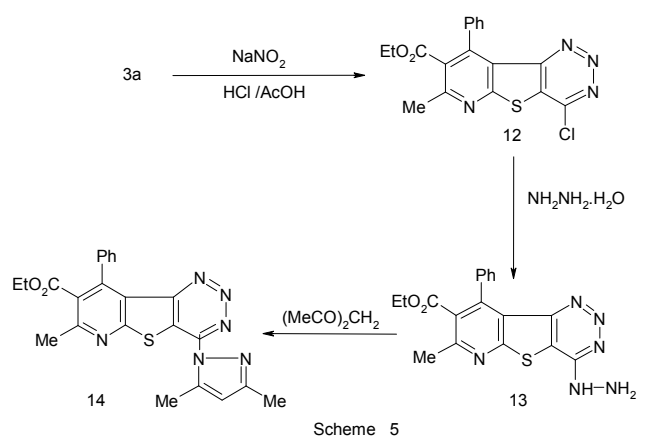

The reaction of $3 \mathrm{a}$ with ethylcyanoacetate in the presence of a catalyticamount of dimethyl formamide gave 8-ethoxycarbonyl-9-methyl-5- 
oxo-7-phenylpyrazolo[3",4"-c]pyrido $\left[3^{\prime}, 2 ': 4,5\right]$ thieno[2,3-e]pyridine (15). When the latter compound reacted with 1,4-dioxan containing amounts of triethylamine produced 8-ethoxycarbonyl-9-methyl-5-oxo-7-phenyl pyrazolo[3",4"-c]pyrido[3',2':4,5] thieno[2,3-e] pyridine (16). (Scheme 6).

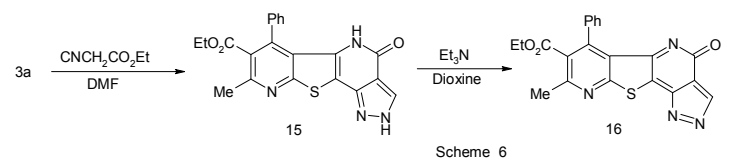

The reaction of 15 with benzaldehyde produced the fused banzoylmethylpyrazolo pyridothienopyridine derivative 17 . Treatment of 15 with active methylene as ethylcyano acetate, in 1,4-dioxan containing catalytic amounts of triethylamine produced amino pyrrolopyrazolopyridothienopyridines 18 . Also, the interaction of compound 15 with phenylisothiocyanate in 1,4-dioxane containing catalytic amounts of triethylamine gave 8ethoxycarbonyl-9-methyl-5-oxo-7-phenyl-3(phenylthiomethylamide)pyrazolo $\quad[3 ", 4 "-$ c]pyrido[3',2':4,5] thieno[2,3-e]pyridine (19). (Scheme 7).

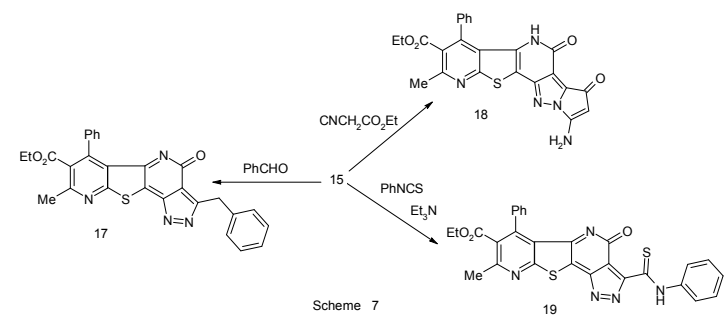

In the same manner, the reaction of compound 15 with ethyl cyanoacetate or phenylisothiocyanate and elemental sulfur in 1,4-dioxane containing triethylamine produced pyridothienopyridine derivative 20 and pyrazolopyridothienopyridine derivative 21 respectively. Finally, compound 15 was reacted with benzenediazonium chloride to give 8-ethoxycarbonyl-9-methyl-5-oxo-7phenyl-3-phenylazinopyrazolo[3",4"-c]pyrido $\left[3^{\prime}, 2^{\prime}: 4,5\right]$ thieno[2,3-e]pyridine (22). (Scheme $8)$.

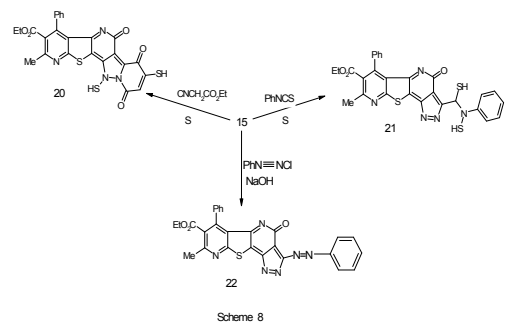

3- EXPERIMENTAL

The uncorrected melting points were determined on a thermometer $360{ }^{\circ} \mathrm{C}$. The IR spectrum were recorded on a VAR AM-2000 FT-IR scimitar series. ${ }^{1} \mathrm{H}$-NMR spectrum were recorded with operating at 500 and $200 \mathrm{MHz}$ respectively using a JEOL ECP400 NMR meter. ${ }^{13} \mathrm{C}$-NMR spectrum were determined on DEITA-NMR ECP-400 400MHz spectrometer with tetramethylsilane (TMS). Mass spectrum were taken on Ionization Mode: EI, $70 \mathrm{eV}$ and checking the homogeneity of the compounds were made by TLC (thin layer chromatography).

Synthesis of 4-aryl-3-cyano-2-cyanomethyl sulfanyl-5-ethoxycarbonyl-6-methylpyridine

$2 a, b$.

To a suspended 4-aryl-3-cyano-5-ethoxy carbonyl-6-methylpyridine-2-(1H)-thiones $1 \mathrm{a}, \mathrm{b}$ (20 mmol) and sodium acetate tri hydrate ( $3 \mathrm{~g}$, $22 \mathrm{mmol})$ in ethanol $(50 \mathrm{ml})$, chloro acetonitrile $(20 \mathrm{mmol})$ was added. The resulting mixture was heated under reflux for $2 \mathrm{~h}$. The precipitate that formed on cooling was collected and recrystallized from ethanol as pale yellow needles of compounds $2 \mathrm{a}, \mathrm{b}$.

\section{3-Cyano-2-cyanomethylsulfanyl-5-}

ethoxycarbonyl-6-methyl-4-phenylpyridin $2 a$.

Prepared from 1a. Yield: $80 \%$; m.p: $120{ }^{\circ} \mathrm{C}$.

Anal. for $\mathrm{C}_{18} \mathrm{H}_{15} \mathrm{~N}_{3} \mathrm{O}_{2} \mathrm{~S}$ (337.40) Calcd.: C, 64.08; H, 4.48; N,12.45 \%. Found: C, 64.12; H, 4.36; N,12.28 \%. The IR 9a showed two 
absorption bands at 2230, $2220 \mathrm{~cm}^{-1}$ for (2 $\mathrm{C} \equiv \mathrm{N})$ and at $1735 \mathrm{~cm}^{-1}$ for $(\mathrm{C}=\mathrm{O}$ of ester $)$. The ${ }^{1} \mathrm{H}$ NMR (DMSO-d ${ }_{6}$ ) at $\delta=7.4-7.6(\mathrm{~m}, 5 \mathrm{H}$, aromatic protons), $4.0\left(\mathrm{q}, 2 \mathrm{H}, \mathrm{OCH}_{2}\right), 3.8(\mathrm{~s}$, $\left.2 \mathrm{H}, \mathrm{SCH}_{2}\right), 2.6\left(\mathrm{~s}, 3 \mathrm{H}, \mathrm{CH}_{3}\right.$ at $\left.\mathrm{C}_{6}\right)$ and $0.9(\mathrm{t}$, $3 \mathrm{H}, \mathrm{CH}_{3}$ of ester) with disappearance a signal for $(\mathrm{NH}) .{ }^{13} \mathrm{C}$ NMR $\left(\mathrm{DMSO}_{-} \mathrm{d}_{6}\right.$ ) at $\delta=169$ $\left(\mathrm{C}_{\text {amide, }} \mathrm{C}=\mathrm{O}\right), 167\left(\mathrm{C}_{\mathrm{ester}}, \mathrm{C}=\mathrm{O}\right), 148\left(\mathrm{C}_{\mathrm{py}}, \mathrm{C}_{4}\right)$, $138\left(\mathrm{C}_{\mathrm{ph}}, \mathrm{C}_{1}\right), 137\left(\mathrm{C}_{\mathrm{py}}, \mathrm{C}_{6}\right), 128,129\left(\mathrm{C}_{\mathrm{ph}}\right.$, $\left.\mathrm{C}_{2,3,4,5,6}\right), 120\left(\mathrm{C}_{\mathrm{py}}, \mathrm{C}_{5}\right), 118(\mathrm{CN}), 98\left(\mathrm{C}_{\mathrm{py}}, \mathrm{C}_{3}\right)$, $60\left(\mathrm{C}, \mathrm{OCH}_{2}\right), 14\left(\mathrm{C}, \mathrm{ArCH}_{3}\right)$ and $13\left(\mathrm{C}, \mathrm{CH}_{3}\right)$. The mass at $\mathrm{m} / \mathrm{z}=337\left(\mathrm{M}^{+}, 60 \%\right)$ and 308 $\left(\mathrm{M}^{+}-29,100 \%\right)$.

\section{4-(4-Chlolrophenyl)-3-cyano-2-cyanomethyl} sulfanyl-5-ethoxycarbonyl-6-methylpyridine $2 b$.

Prepared from 1b. Yield: 64 \%; m.p: $130{ }^{\circ} \mathrm{C}$. Anal. Calcd. for $\mathrm{C}_{18} \mathrm{H}_{14} \mathrm{ClN}_{3} \mathrm{O}_{2} \mathrm{~S}$ (371.85): C, 58.14; H, 3.79; N, $11.30 \%$. Found: C, 58.01; $\mathrm{H}, 3.58 ; \mathrm{N}, 11.24 \%$. IR $\left(\mathrm{cm}^{-1}\right)$ at 2230,2220 for $(2 \mathrm{C} \equiv \mathrm{N})$ and at 1735 for $(\mathrm{C}=\mathrm{O}$ of ester $) .{ }^{1} \mathrm{H}$ NMR at $\delta=7.3-7.5$ (m, $4 \mathrm{H}$, aromatic protons), $4\left(\mathrm{q}, 2 \mathrm{H}, \mathrm{OCH}_{2}\right), 3.8\left(\mathrm{~s}, 2 \mathrm{H}, \mathrm{SCH}_{2}\right), 2.6(\mathrm{~s}, 3 \mathrm{H}$, $\mathrm{CH}_{3}$ at $\mathrm{C}_{6}$ ) and 0.9 (t, $3 \mathrm{H}, \mathrm{CH}_{3}$ of ester). ${ }^{13} \mathrm{C}$ NMR (DMSO-d $\left.{ }_{6}\right)$ at $\delta=169\left(\mathrm{C}_{\text {amide, }} \mathrm{C}=\mathrm{O}\right)$, $167\left(\mathrm{C}_{\mathrm{ester}}, \mathrm{C}=\mathrm{O}\right), 162\left(\mathrm{C}_{\mathrm{py}}, \mathrm{C}_{6}\right), 152148\left(\mathrm{C}_{\mathrm{py}}\right.$, $\left.\mathrm{C}_{4}\right), 146\left(\mathrm{C}_{\mathrm{th}}, \mathrm{C}_{2}\right), 138\left(\mathrm{C}_{\mathrm{ph},}, \mathrm{C}_{1}\right), 134\left(\mathrm{C}_{\mathrm{ph}}, \mathrm{C}_{4}\right)$, 128, $129\left(\mathrm{C}_{\mathrm{ph}}, \mathrm{C}_{2,3,5,6}\right), 120\left(\mathrm{C}_{\mathrm{py}}, \mathrm{C}_{5}\right), 118(\mathrm{CN})$, $98\left(\mathrm{C}_{\mathrm{py}}, \mathrm{C}_{3}\right), 60\left(\mathrm{C}, \mathrm{OCH}_{2}\right), 14\left(\mathrm{C}, \mathrm{ArCH}_{3}\right)$ and $13\left(\mathrm{C}, \mathrm{CH}_{3}\right)$. Mass at $\mathrm{m} / \mathrm{z}=370\left(\mathrm{M}^{+}-1,100 \%\right)$.

Synthesis of 3-amino-4-aryl-2-carbamoyl-5ethoxycarbonyl-6-methylthieno[2,3-b] pyridine $3 a, b$.

\section{Method A}

Compounds 2a,b were suspended in sodium ethoxide solution $(0.12 \mathrm{~g}$ sodium in $30 \mathrm{ml}$ abs. ethanol) and heated under reflux for $5 \mathrm{~min}$. The solid that formed while hot was collected and recrystallized from ethanol-chloroform mixture to give canary yellow needles of compounds $415 \mathrm{a}, \mathrm{b}$.

3-Amino-2-carbamoyl-5-ethoxycarbonyl-6methyl-4-phenylthieno[2,3-b]pyridine 3a.

Prepared from 2a. Yield: $81 \%$; m.p: $300{ }^{\circ} \mathrm{C}$. Anal. Calcd. for $\mathrm{C}_{18} \mathrm{H}_{17} \mathrm{~N}_{3} \mathrm{O}_{3} \mathrm{~S}$ (355.42): C, $60.83 ; \mathrm{H}, 4.82$; N, $11.82 \%$. Found: C, 60.59; $\mathrm{H}, 4.68 ; \mathrm{N}, 11.71 \%$. IR $\left(\mathrm{cm}^{-1}\right)$ at 3440,3360 for $\left(\mathrm{NH}_{2}\right), 1700$ for $(\mathrm{C}=\mathrm{O}$ groups $)$ with disappearance absorption bands for $(\mathrm{C} \equiv \mathrm{N}) .{ }^{1} \mathrm{H}$ NMR $\left(\mathrm{CDCl}_{3}\right)$ at $\delta=6.9-7.5(\mathrm{~m}, 7 \mathrm{H}$, aromatic and amid protons), $4.2(\mathrm{q}, 2 \mathrm{H}$, $\left.\mathrm{OCH}_{2}\right), 3.8\left(\mathrm{~s}, 2 \mathrm{H}, \mathrm{NH}_{2}\right), 2.3\left(\mathrm{~s}, 3 \mathrm{H}, \mathrm{CH}_{3}\right.$ at $\left.\mathrm{C}_{6}\right)$ and 0.9 (t, $3 \mathrm{H}, \mathrm{CH}_{3}$ of ester) with disappearance a signal for $\left.\left(\mathrm{SCH}_{2}\right).\right) \cdot{ }^{13} \mathrm{C}$ $\operatorname{NMR}\left(\mathrm{CDCl}_{3}\right)$ at $\delta=169\left(\mathrm{C}_{\text {amide, }} \mathrm{C}=\mathrm{O}\right), 167$ $\left(\mathrm{C}_{\text {ester, }} \mathrm{C}=\mathrm{O}\right), 158\left(\mathrm{C}_{\mathrm{py}}, \mathrm{C}_{6}\right), 148\left(\mathrm{C}_{\mathrm{py}}, \mathrm{C}_{4}\right), 146$ $\left(\mathrm{C}_{\mathrm{th}}, \mathrm{C}_{2}\right), 136\left(\mathrm{C}_{\mathrm{ph}}, \mathrm{C}_{1}\right), 137\left(\mathrm{C}_{\mathrm{th}}, \mathrm{C}_{3}\right), 134\left(\mathrm{C}_{\mathrm{ph}}\right.$, $\left.\mathrm{C}_{4}\right), 128,129\left(\mathrm{C}_{\mathrm{ph}}, \mathrm{C}_{2,3,5,6}\right), 120\left(\mathrm{C}_{\mathrm{py}}, \mathrm{C}_{5}\right), 60$ $\left(\mathrm{C}, \mathrm{OCH}_{2}\right), 14\left(\mathrm{C}, \mathrm{ArCH}_{3}\right)$ and $13\left(\mathrm{C}, \mathrm{CH}_{3}\right)$. Mass at $\mathrm{m} / \mathrm{z}=337\left(\mathrm{M}^{+}-18,100 \%\right)$.

\section{3-Amino-2-carbamoyl-4-(4-chlolrophenyl)-5-}

ethoxycarbonyl-6-methylthieno[2,3-b]

pyridine $3 b$.

Prepared from 2b.Yield: $80 \%$; m.p: $320{ }^{\circ} \mathrm{C}$. Anal. Calcd. for $\mathrm{C}_{18} \mathrm{H}_{16} \mathrm{ClN}_{3} \mathrm{O}_{3} \mathrm{~S}$ (389.64): C, 55.46; H, 4.14; N, 10.78 \%.Found: C, 55.32 ; $\mathrm{H}, 3.99 ; \mathrm{N}, 10.53 \%$. IR $\left(\mathrm{cm}^{-1}\right)$ at 3440,3360 for $\left(\mathrm{NH}_{2}\right), 1700$ for $(\mathrm{C}=\mathrm{O}$ groups $)$ with disappearance absorption bands for $(\mathrm{C} \equiv \mathrm{N}) .{ }^{1} \mathrm{H}$ NMR $\left(\mathrm{CDCl}_{3}\right)$ at $\delta=7.1-7.7(\mathrm{~m}, 6 \mathrm{H}$, aromatic and amid protons), $3.9-4.1$ (q\&s, $\left.4 \mathrm{H}, \mathrm{OCH}_{2}, \mathrm{NH}_{2}\right), 2.7\left(\mathrm{~s}, 3 \mathrm{H}, \mathrm{CH}_{3}\right.$ at $\left.\mathrm{C}_{6}\right)$ and $1.0\left(\mathrm{t}, 3 \mathrm{H}, \mathrm{CH}_{3}\right.$ of ester). Mass at $\mathrm{m} / \mathrm{z}=371$ $\left(\mathrm{M}^{+}, 95 \%\right)$ and $190\left(\mathrm{M}^{+}-181,100 \%\right)$.

\section{Method B}


To a suspension of compounds $2 \mathrm{a}, \mathrm{b}$ in sodium ethoxide solution $(0.35 \mathrm{~g}$ sodium in 40 $\mathrm{ml}$ abs. ethanol), chloro acetonitrile (10 mmol) was added. The resulting mixture was refluxed for $20 \mathrm{~min}$. The formed precipitate was collected and recrystallized from ethanolchloroform mixture to give compounds $3 \mathrm{a}, \mathrm{b}$ (yield; 57 - $60 \%$ ). These products were identical in all aspects to those described in method A.

Synthesis of 3-amino-2-(4,5-dihydro imidazole-2-yl)-5-ethoxycarbonyl-6-methyl-4phenylthieno[2,3-b]pyridine (4).

To a suspension of compound $3 \mathrm{a}$ (1.6 g, 5 $\mathrm{mmol})$ and ethylenediamine $(5 \mathrm{ml})$ were heated on a water bath for $5 \mathrm{~h}$. and then triturated with ethanol $(10 \mathrm{ml})$. The solid that formed was collected and recrystallized from ethanolchloroform mixture to give golden yellow crystals. Yield: $80 \%$; m.p: $170{ }^{\circ} \mathrm{C}$. Anal. Calcd. for $\mathrm{C}_{20} \mathrm{H}_{20} \mathrm{~N}_{4} \mathrm{O}_{2} \mathrm{~S}$ (380.46): C, 63.14; $\mathrm{H}$, $5.30 ; \mathrm{N}, 14.73$ \%. Found: C, 63.25; H, 5.19; N, $14.54 \%$. IR $\left(\mathrm{cm}^{-1}\right)$ at 3400,3320 for $\left(\mathrm{NH}_{2}\right.$, $\mathrm{NH})$ and 1720 for $(\mathrm{C}=\mathrm{O}$ ester $) .{ }^{1} \mathrm{H}$ NMR $\left(\right.$ DMSO- $\left._{6}\right)$ at $\delta=7.2-7.5(\mathrm{~m}, 5 \mathrm{H}$, aromatic protons), 4.1(s, $3 \mathrm{H}, \mathrm{NH}$ and $\left.\mathrm{NH}_{2}\right), 4.0(\mathrm{q}, 2 \mathrm{H}$, $\mathrm{OCH}_{2}$ ), 2.7 (t, $2 \mathrm{H}, \mathrm{CH}_{2}$ imidazolo), 2.4 (s, 3H, $\mathrm{CH}_{3}$ at $\left.\mathrm{C}_{6}\right), 1.4\left(\mathrm{t}, 2 \mathrm{H}, \mathrm{CH}_{2}\right.$ imidazolo) and 0.9 $\left(\mathrm{t}, 3 \mathrm{H}, \mathrm{CH}_{3}\right.$ of ester). ${ }^{13} \mathrm{C}$ NMR (DMSO- $\left.\mathrm{d}_{6}\right)$ at $\delta$ $\left.=167\left(\mathrm{C}_{\text {ester, }}, \mathrm{C}=\mathrm{O}\right), 165 \mathrm{C}_{\text {imid, }} \mathrm{C}_{2}\right), 158\left(\mathrm{C}_{\mathrm{py}}\right.$, $\left.\mathrm{C}_{6}\right), 155\left(\mathrm{C}_{\mathrm{py}}, \mathrm{C}_{3}\right), 148\left(\mathrm{C}_{\mathrm{py}}, \mathrm{C}_{4}\right), 126\left(\mathrm{C}_{\mathrm{ph}}\right.$, $\left.\mathrm{C}_{2,334,5,6}\right), 127\left(\mathrm{C}_{\mathrm{th}}, \mathrm{C}_{3,4}\right), 126\left(\mathrm{C}_{\mathrm{th}}, \mathrm{C}_{2,5}\right), 60(\mathrm{C}$, $\left.\mathrm{OCH}_{2}\right), 53\left(\mathrm{C}_{\text {imid, }} \mathrm{C}_{4}\right), 35\left(\mathrm{C}_{\text {imid, }} \mathrm{C}_{5}\right), 129,14$ $\left(\mathrm{C}, \mathrm{ArCH}_{3}\right)$ and $13\left(\mathrm{C}, \mathrm{CH}_{3}\right)$. Mass at $\mathrm{m} / \mathrm{z}=380$ $\left(\mathrm{M}^{+}, 70 \%\right)$ and $54\left(\mathrm{M}^{+}-326,100 \%\right)$.

Synthesis of 3-amino-5-ethoxycarbonyl-6methyl-4-phenyl-2-(1H)-tetrazol-2-yl)

thieno[2,3-b]pyridine (5).
A mixture of compound $3 \mathrm{a}(1.4 \mathrm{~g}, 4 \mathrm{mmol})$, sodium azide $(0.4 \mathrm{~g}, 6 \mathrm{mmol})$ and ammonium chloride (0.32 g, $6 \mathrm{mmol})$ in DMF (15 ml) was heated on a water bath for $2 \mathrm{~h}$. The reaction mixture was cooled, diluted with water and acidified with dilute acetic acid. The solid that formed was collected and recrystallized from ethanol to give golden yellow crystals. Yield: 75 \%; m.p: $312{ }^{\circ} \mathrm{C}$. Anal. Calcd. for $\mathrm{C}_{18} \mathrm{H}_{16} \mathrm{~N}_{6} \mathrm{O}_{2} \mathrm{~S}$ (380.42): C, 56.83; H, 4.24; N, $22.09 \%$. Found: C, 56.64; H, 4.06; N, $22.15 \%$. IR $\left(\mathrm{cm}^{-1}\right)$ at 3430,3350 for $\left(\mathrm{NH}_{2}\right.$, $\mathrm{NH})$ and $1720 \mathrm{~cm}^{-1}$ for ( $\mathrm{C}=\mathrm{O}$ ester). ${ }^{1} \mathrm{H}$ NMR $\left(\mathrm{CDCI}_{3}\right)$ at $\delta=8.0$ (s, 1H, NH tetrazolo), 7.1 7.5 (m, 5H, aromatic protons), $3.9-4.1$ (s \& q, $\left.4 \mathrm{H}, \mathrm{NH}_{2} \& \mathrm{OCH}_{2}\right), 2.4\left(\mathrm{~s}, 3 \mathrm{H}, \mathrm{CH}_{3}\right.$ at $\left.\mathrm{C}_{6}\right)$ and $0.8\left(\mathrm{t}, 3 \mathrm{H}, \mathrm{CH}_{3}\right.$ of ester). Mass at $\mathrm{m} / \mathrm{z}=380$ $\left(\mathrm{M}^{+}, 45 \%\right)$ and $120\left(\mathrm{M}^{+}-260,100 \%\right)$.

Synthesis of 8-ethoxycarbonyl-9-methyl-7phenyl-2,3-dihydroimidazolo[1",2"-c]pyrido $\left[3^{\prime}, 2^{\prime}: 4,5\right]$ thieno[2,3-e]pyrimidine (6).

Compound $4(0.76 \mathrm{~g}, 2 \mathrm{mmol})$ in triethylorthoformate $(10 \mathrm{ml})$ was heated under reflux for $4 \mathrm{~h}$. The precipitate that formed while hot was collected and recrystallized from pyridine to give of pale yellow needles. Yield: $80 \%$; m.p: $285{ }^{\circ} \mathrm{C}$. Anal. Calcd. for $\mathrm{C}_{21} \mathrm{H}_{18} \mathrm{~N}_{4} \mathrm{O}_{2} \mathrm{~S}$ (390.45): C, 64.59; H, 4.64; N, 14.34 \%. Found: C, 64.47; H, 4.45; N, 14.28 $\%$. IR $\left(\mathrm{cm}^{-1}\right)$ at 3050 for $\left(=\mathrm{CH}_{\text {aromatic }}\right), 2950$ for $\left(\mathrm{CH}_{\text {aliphatic }}\right), 1718$ for $(\mathrm{C}=\mathrm{O}$ of ester $)$ and 1620 for $\left(\mathrm{C}=\mathrm{C}_{\text {aromatic }}\right) .{ }^{1} \mathrm{H}$ NMR $\left(\mathrm{CDCl}_{3}\right)$ at $\delta=7.1-$ $7.5(\mathrm{~m}, 7 \mathrm{H}$, aromatic and $\mathrm{CH}$ pyrazolo protons), 4.0 (q, 2H, $\left.\mathrm{OCH}_{2}\right), 2.8$ (t, $2 \mathrm{H}, \mathrm{CH}_{2}$ imidazolo), 2.4 (s, $3 \mathrm{H}, \mathrm{CH}_{3}$ at $\left.\mathrm{C}_{9}\right), 1.4(\mathrm{t}, 2 \mathrm{H}$, $\mathrm{CH}_{2}$ imidazolo) and $1.0\left(\mathrm{t}, 3 \mathrm{H}, \mathrm{CH}_{3}\right.$ of ester). Mass at $\mathrm{m} / \mathrm{z}=390\left(\mathrm{M}^{+}, 80 \%\right)$ and $341\left(\mathrm{M}^{+}\right.$$49,100 \%)$. 
Synthesis of 5-(4-chloropyenyl)-8-ethoxy carbonyl-9-methyl-7-phenyl-2,3,5,6-tetra

hydroimidazolo[1",2"-c]pyrido[3',2':4,5]

thieno[2,3-e]pyrimidine (7).

A suspension of compound $4(0.76 \mathrm{~g}, 2$ mmol) and 4-chloro benzaldehyde (0.2 g, 2 mmol) in ethanol $(15 \mathrm{ml})$, few drops of in piperidine was added. The reaction mixture was heated under reflux for $4 \mathrm{~h}$. The solid that formed was on cooling collected and recrystallized from ethanol was collected and recrystallized from dioxane to give yellow crystals. Yield: $81 \%$; m.p:312 ${ }^{\circ} \mathrm{C}$. Anal. Calcd. for $\mathrm{C}_{27} \mathrm{H}_{23} \mathrm{ClN}_{4} \mathrm{O}_{2} \mathrm{~S}$ (503.01): C, 64.46; $\mathrm{H}$, 4.60 ; N, $11.13 \%$. Found: C, 64.28; H, 3.46; $\mathrm{N}, 11.00 \%$. The IR $\left(\mathrm{cm}^{-1}\right)$ at $3400-3350$ for $\mathrm{NH}$ and 1720 for $\left(\mathrm{C}=\mathrm{O}\right.$ of ester). ${ }^{1} \mathrm{H}$ NMR $\left(\mathrm{CDCl}_{3}\right)$ at $\delta=8.0$ (s, 1 $\mathrm{H}, \mathrm{NH}$ pyrimidine $), 7.1$ - $7.6(\mathrm{~m}, 11 \mathrm{H}$, aromatic and $\mathrm{CH}$ pyrazolo protons), 4.0 (q, $2 \mathrm{H}, \mathrm{OCH}_{2}$ ), 2.7 (t, $2 \mathrm{H}, \mathrm{CH}_{2}$ imidazolo), 2.4 (s, $3 \mathrm{H}, \mathrm{CH}_{3}$ at $\mathrm{C}_{9}$ ), $1.4(\mathrm{t}, 2 \mathrm{H}$, $\mathrm{CH}_{2}$ imidazolo) and 0.9 (t, 3H, $\mathrm{CH}_{3}$ of ester). ${ }^{13} \mathrm{C}$ NMR $\left(\right.$ DMSO-d $\left._{6}\right)$ at $\delta=167\left(\mathrm{C}_{\text {ester, }} \mathrm{C}=\mathrm{O}\right)$, $165\left(\mathrm{C}_{\mathrm{py}}, \mathrm{C}_{2}\right), 158\left(\mathrm{C}_{\mathrm{py}}, \mathrm{C}_{6}\right), 148\left(\mathrm{C}_{\mathrm{py}}, \mathrm{C}_{4}\right), 138$ $\left(\mathrm{C}_{\mathrm{ph}}, \mathrm{C}_{1}\right), 134\left(\mathrm{C}_{\mathrm{ph}}, \mathrm{C}_{4}\right), 129\left(\mathrm{C}_{\mathrm{ph}}, \mathrm{C}_{2,3,5,5}\right), 126$ $\left(\mathrm{C}_{\mathrm{py}}, \mathrm{C}_{5,6}\right), 126\left(\mathrm{C}_{\mathrm{th}}, \mathrm{C}_{2,3}\right), 122\left(\mathrm{C}_{\text {imid, }}, \mathrm{C}_{4,5}\right), 60$ $\left(\mathrm{C}, \mathrm{OCH}_{2}\right), 14\left(\mathrm{C}, \mathrm{ArCH}_{3}\right)$ and $13\left(\mathrm{C}, \mathrm{CH}_{3}\right)$. Mass at $\mathrm{m} / \mathrm{z}=505\left(\mathrm{M}^{+}+2,20 \%\right)$ and $149\left(\mathrm{M}^{+}\right.$ $-349,100 \%)$.

Synthesis of 8-ethoxycarbonyl-9-methyl-7phenyl-5-thioxo-2,3dihydroimidazolo $[1 ", 2$ "clpyrido[3',2':4,5]thieno[2,3-e]pyrimidine (8).

A mixture of compound 4 (0.76 g, $2 \mathrm{mmol})$ and carbon disulphide $(1 \mathrm{ml})$ in pyridine $(10$ $\mathrm{ml}$ ) was heated under reflux on a water bath for $8 \mathrm{~h}$. The solid that formed while hot was collected and recrystallized from DMF to give orange crystals. Yield: $65 \%$ m.p: $240{ }^{\circ} \mathrm{C}$.
Anal. Calcd. for $\mathrm{C}_{21} \mathrm{H}_{18} \mathrm{~N}_{4} \mathrm{O}_{2} \mathrm{~S}_{2}$ (422.52): C, 59.69; H, 4.28; N, 13.25 \%. Found: C, 59.46; $\mathrm{H}, 4.19 ; \mathrm{N}, 13.16 \%$. IR $\left(\mathrm{cm}^{-1}\right)$ at $3400-3350$ for $\mathrm{NH}$ and 1720 for $\left(\mathrm{C}=\mathrm{O}\right.$ of ester). ${ }^{1} \mathrm{H}$ NMR $\left(\mathrm{CDCl}_{3}\right)$ at $\delta=8.0$ (s, $1 \mathrm{H}, \mathrm{NH}$ pyrimidine), 7.1 - $7.9(\mathrm{~m}, 7 \mathrm{H}$, aromatic and $\mathrm{CH}$ pyrazolo protons), 4.1 (s, 1H, NH), 3.9 (q, $2 \mathrm{H}, \mathrm{OCH}_{2}$ ), 2.7 (t, $2 \mathrm{H}, \mathrm{CH}_{2}$ imidazolo), 2.5 (s, 3H, $\mathrm{CH}_{3}$ at $\left.\mathrm{C}_{9}\right), 1.4\left(\mathrm{t}, 2 \mathrm{H}, \mathrm{CH}_{2}\right.$ imidazolo) and $1.0(\mathrm{t}, 3 \mathrm{H}$, $\mathrm{CH}_{3}$ of ester). Mass at $\mathrm{m} / \mathrm{z}=422\left(\mathrm{M}^{+}, 95 \%\right)$ and $341\left(\mathrm{M}^{+}-79,100 \%\right)$.

Synthesis of 8-ethoxycarbonyl-9-methyl-7phenyl-2,3-dihydroimidazolo[1",2"-c]pyrido $\left[3^{\prime}, 2^{\prime}: 4,5\right]$ thieno[2,3-e][1,2,3]triazine (9).

Sodium nitrite solution $10 \%(5 \mathrm{ml})$ was added to a solution of compound 4 ( $0.76 \mathrm{~g}, 2$ mmol) in concentrated sulphuric acid $(5 \mathrm{ml})$ and glacial acetic acid $(5 \mathrm{ml})$ at $0{ }^{\circ} \mathrm{C}$ during 5 min. with stirring. The mixture was allowed to stand at room temperature for $30 \mathrm{~min}$. The solid that precipitated on dilution with water was collected and recrystallized from ethanol as white needles. Yield: $72 \%$; m.p: $150{ }^{\circ} \mathrm{C}$. Anal. Calcd. for $\mathrm{C}_{20} \mathrm{H}_{17} \mathrm{~N}_{5} \mathrm{O}_{2} \mathrm{~S}$ (391.44): C, 61.36; H, 4.37; N, 17.88 \%. Found: C, 61.23; $\mathrm{H}, 4.14 ; \mathrm{N}, 17.57 \%$. IR $\left(\mathrm{cm}^{-1}\right)$ at 1720 for $(\mathrm{C}=\mathrm{O}$ of ester) with the disappearance band of $\left(\mathrm{NH}_{2}\right) .{ }^{1} \mathrm{H}$ NMR $\left(\mathrm{CDCl}_{3}\right)$ at $\delta=7.1-7.5(\mathrm{~m}$, $7 \mathrm{H}$, aromatic and $\mathrm{CH}$ pyrazolo protons), 4.0 (q, $2 \mathrm{H}, \mathrm{OCH}_{2}$ ), 2.7 (t, $2 \mathrm{H}, \mathrm{CH}_{2}$ imidazolo), 2.5 (s, $3 \mathrm{H}, \mathrm{CH}_{3}$ at $\left.\mathrm{C}_{9}\right), 1.4\left(\mathrm{t}, 2 \mathrm{H}, \mathrm{CH}_{2}\right.$ imidazolo) and 0.9 (t, $3 \mathrm{H}, \mathrm{CH}_{3}$ of ester). Mass at $\mathrm{m} / \mathrm{z}=389$ $\left(\mathrm{M}^{+}, 15 \%\right)$ and $256\left(\mathrm{M}^{+}-133,100 \%\right)$.

Synthesis of 8-ethoxycarbonyl-9-methyl-7phenyltetrazolo[1",2"-c]pyrido[3',2':4,5]

thieno[2,3-e]pyrimidine (10).

Compound $5 \quad(0.76 \quad \mathrm{~g}, \quad 2 \mathrm{mmol})$ in triethylorthoformate $(10 \mathrm{ml})$ was heated under 
reflux for $3 \mathrm{~h}$. and then left to cool. The precipitate that formed was collected and recrystallized from dioxane to give of pale yellow needles. Yield: 60\%; m.p: $180{ }^{\circ} \mathrm{C}$. Anal. Calcd. for $\mathrm{C}_{19} \mathrm{H}_{14} \mathrm{~N}_{6} \mathrm{O}_{2} \mathrm{~S}$ (390.42): C, 58.45; H, 3.61; N, 21.53 \%. Found: C, 58.38; H, 3.42; N, $21.46 \%$.

Synthesis of 8-ethoxycarbonyl-6-hydro-9methyl-7-phenyl-5-thioxotetrazolo[1",2"-c] pyrido[3',2':4,5]thieno[2,3-e]pyrimidine (11).

A mixture of compound $5(0.76 \mathrm{~g}, 2 \mathrm{mmol})$ and carbon disulphide $(1 \mathrm{ml})$ in pyridine $(10$ $\mathrm{ml}$ ) was heated under reflux on a water bath for $12 \mathrm{~h}$. The solid that formed while hot was collected and recrystallized from DMF to give yellow crystals. Yield: $79 \%$; m.p: $330{ }^{\circ} \mathrm{C}$. Anal. Calcd. for $\mathrm{C}_{19} \mathrm{H}_{14} \mathrm{~N}_{6} \mathrm{O}_{2} \mathrm{~S}_{2}$ (422.48): C, 54.01; H, 3.34; N,19.89 \%. Found: C, 54.21; H, 3.19; N, $19.69 \%$. The IR $\left(\mathrm{cm}^{-1}\right)$ at 1720 for $\left(\mathrm{C}=\mathrm{O}\right.$ of ester). ${ }^{1} \mathrm{H}$ NMR $\left(\mathrm{DMSO}^{\mathrm{d}} \mathrm{d}_{6}\right)$ at $\delta=8.0$ (s, 1H, CH pyrimidine), $6.7-7.5(\mathrm{~m}, 5 \mathrm{H}$, aromatic protons), $4.0\left(\mathrm{q}, 2 \mathrm{H}, \mathrm{OCH}_{2}\right)$ and 2.4 (s, 3H, $\mathrm{CH}_{3}$ at $\mathrm{C}_{9}$ ) and $1.0\left(\mathrm{t}, 3 \mathrm{H}, \mathrm{CH}_{3}\right.$ of ester). Mass at m/z= $390\left(\mathrm{M}^{+}, 60 \%\right)$ and $97\left(\mathrm{M}^{+}\right.$293, $100 \%$ ).

Synthesis of 4-chloro-8-ethoxycarbonyl-7methyl-9-phenylpyrido[3',2':4,5]thieno[2,3-

e][1,2,3]triazine (12).

To a chilled solution of compound $3 \mathrm{a}(0.76$ $\mathrm{g}, 2 \mathrm{mmol})$ in a mixture of acetic acid $(10 \mathrm{ml})$ and concentrated hydrochloric acid $(7 \mathrm{ml})$, a sodium nitrite solution $10 \%(4 \mathrm{ml})$ was added with stirring at $0{ }^{\circ} \mathrm{c}$ during $5 \mathrm{~min}$. The stirring was continuing for $3 \mathrm{~h}$. The formed precipitated was collected and recrystallized from ethanol-chloroform mixture to give white plated. Yield: $72 \%$; m.p: $200{ }^{\circ} \mathrm{C}$. Anal. Calcd. for $\mathrm{C}_{18} \mathrm{H}_{13} \mathrm{ClN}_{4} \mathrm{O}_{2} \mathrm{~S}$ (384.84): C, 56.18; H, 3.40
; N,14.56 \%. Found: C, 55.88; H, 3.26; $\mathrm{N}, 14.33 \%$. IR $\left(\mathrm{cm}^{-1}\right)$ at $3420-3330$ for $(\mathrm{NH})$ and 1712 for $(\mathrm{C}=\mathrm{O}$ of ester $) .{ }^{1} \mathrm{H} \mathrm{NMR}\left(\mathrm{CDCI}_{3}\right)$ at $\delta=6.8-7.8(5 \mathrm{H}$, aromatic protons), 4.1 4.0 ( s \& q, 3H, NH, $\mathrm{OCH}_{2}$ ), $\delta 2.3\left(\mathrm{~s}, 3 \mathrm{H}, \mathrm{CH}_{3}\right.$ at $\left.\mathrm{C}_{9}\right)$ and $1.0\left(\mathrm{t}, 3 \mathrm{H}, \mathrm{CH}_{3}\right.$ of ester). The mass at $\mathrm{m} / \mathrm{z}=421\left(\mathrm{M}^{+}-1,60 \%\right)$ and $80\left(\mathrm{M}^{+}-342\right.$, $100 \%)$.

Synthesis of 8-ethoxycarbonyl-4-hydrazino 7-methyl-9-phenylpyrido[3',2':4,5]thieno [2,3e][1,2,3]triazine (13).

A mixture of compound $12(0.76 \mathrm{~g}, 2$ mmol) and hydrazine hydrate 99\% $(4 \mathrm{ml})$ in ethanol $(30 \mathrm{ml})$ was heated under reflux for 4 h. The solid that formed while hot was collected and recrystallized from dioxane to give yellow crystals. Yield: $70 \%$; m.p: $240{ }^{\circ} \mathrm{C}$. Anal. Calcd. for $\mathrm{C}_{18} \mathrm{H}_{16} \mathrm{~N}_{6} \mathrm{O}_{2} \mathrm{~S}$ (380.42): C, 56.83; H, 4.24; N, 22.09 \%. Found: C, 56.62; $\mathrm{H}, 4.09 ; \mathrm{N}, 22.21 \%$. IR $\left(\mathrm{cm}^{-1}\right)$ at 3450,3400 for $\left(\mathrm{NH}-\mathrm{NH}_{2}\right)$ and $1724(\mathrm{C}=\mathrm{O}$ of ester $) .{ }^{1} \mathrm{H}$ $\mathrm{NMR} \mathrm{CDCI}_{3}$ at $\delta=7.1-7.6(\mathrm{~m}, 5 \mathrm{H}$, aromatic protons), 4.2 - 4.0 (s \& q, 3H, $\mathrm{OCH}_{2}, \mathrm{NH}$ ), 2.6 $\left(\mathrm{s}, 3 \mathrm{H}, \mathrm{CH}_{3}\right.$ at $\left.\mathrm{C} 7\right), 2.0\left(\mathrm{~s}, 2 \mathrm{H}, \mathrm{NH}_{2}\right)$ and $0.9(\mathrm{t}$, $3 \mathrm{H}, \mathrm{CH}_{3}$ of ester). Mass at $\mathrm{m} / \mathrm{z}=380(\mathrm{M}+, 60$ $\%)$ and $366\left(\mathrm{M}^{+}-14,100 \%\right)$.

Synthesis of 4-(3,5-dimethylpyrazolo-1-yl)-8ethoxycarbonyl-7-methyl-9-phenylpyrido $\left[3^{\prime}, 2^{\prime}: 4,5\right]$ thieno[2,3-e][1,2,3]triazine (14).

A compound $13(0.76 \mathrm{~g}, 2 \mathrm{mmol})$ and acetylacetone $(2 \mathrm{ml}, 20 \mathrm{mmol})$ in ethanol (15 $\mathrm{ml}$ ) was heated under reflux for $5 \mathrm{~h}$. The precipitate that formed while hot was collected and recrystallized from ethanolchloroform mixture as greenish crystals of 14 . Yield: $30 \%$; m.p: $320{ }^{\circ} \mathrm{C}$. Anal. Calcd. for $\mathrm{C}_{23} \mathrm{H}_{20} \mathrm{~N}_{6} \mathrm{O}_{2} \mathrm{~S}$ (444.52): C, 62.14; H, 4.53; N,18.79 \%. Found: C, 62.36; H, 4.77; N, 18.46 
\%. IR $\left(\mathrm{cm}^{-1}\right)$ at 1725 for $(\mathrm{C}=\mathrm{O}$ of ester $)$ with disappearance absorption bands of $\left(\mathrm{NH}_{2}\right) .{ }^{1} \mathrm{H}$ NMR $\mathrm{CDCI}_{3}$ at $\delta=7.2-7.6(\mathrm{~m}, 5 \mathrm{H}$, aromatic protons), 4.1 (q, $2 \mathrm{H}, \mathrm{OCH}_{2}$ ), 2.7 (s, $6 \mathrm{H}, 2 \mathrm{CH}_{3}$ pyrazolo), $2.5\left(\mathrm{~s}, 3 \mathrm{H}, \mathrm{CH}_{3}\right.$ at $\left.\mathrm{C}_{7}\right)$ and $0.8(\mathrm{t}$, $3 \mathrm{H}, \mathrm{CH}_{3}$ of ester). Mass at $\mathrm{m} / \mathrm{z}=444\left(\mathrm{M}^{+}, 15\right.$ $\%)$ and $312\left(\mathrm{M}^{+}-123,100 \%\right)$.

Synthesis of 1,6-dihydro-8-ethoxycarbonyl9-methyl-5-oxo-7-phenylpyrazolo[3",4"-c] pyrido[3',2':4,5]thieno[2,3-e] pyridine (15).

A mixture of $3 \mathrm{a}(3.7 \mathrm{~g}, 10 \mathrm{mmol})$ in dimethylformamide $(30 \mathrm{ml})$, ethyl cyanoacetate $(1.1 \mathrm{ml}, 10 \mathrm{mmol})$ was added. The reaction mixture was heated under reflux for $5 \mathrm{~h}$. The solid that formed while hot was collected and recrystallized from dioxane to give pale yellow crystals. Yield: $82 \%$; m.p: $330{ }^{\circ} \mathrm{C}$. Anal. for $\mathrm{C}_{21} \mathrm{H}_{16} \mathrm{~N}_{4} \mathrm{O}_{3} \mathrm{~S}$ (404.44) Calcd.: C, 62.36; $\mathrm{H}$, 3.99 ; N, $13.85 \%$. Found: C, 62.11; H, 3.82; N, $13.67 \%$. IR $\left(\mathrm{cm}^{-1}\right)$ at 3420,3320 for $(2 \mathrm{NH})$, at 1716 for $\left(\mathrm{C}=\mathrm{O}\right.$ groups). ${ }^{1} \mathrm{H}$ NMR $\mathrm{CDCI}_{3}$ at $\delta=$ $8.0-8.1(\mathrm{~s}, 2 \mathrm{H}, \mathrm{NH}$ pyrazolo and $\mathrm{NH}$ pyridine), $7.2-7.6(\mathrm{~m}, 6 \mathrm{H}$, aromatic and $\mathrm{CH}$ pyrazolo protons), 4.0 (q, $\left.2 \mathrm{H}, \mathrm{OCH}_{2}\right), 2.4$ (t, $3 \mathrm{H}, \mathrm{CH}_{3}$ at $\mathrm{C}_{9}$ ) and $\delta 0.8$ (t, $3 \mathrm{H}, \mathrm{CH}_{3}$ at ester). ${ }^{13} \mathrm{C} \mathrm{NMR}\left(\mathrm{CDCI}_{3}\right)$ at $\delta=167($ Cester, $\mathrm{C}=\mathrm{O})$, 165 (Cpy, C=O), 158 (Cpy, C6), 148 (Cpy, C4), 138 (Cph, C1), 133(Cpyr, C C 3,5$), 129$ $\left(\mathrm{C}_{\mathrm{ph}}, \mathrm{C}_{2,3,4,5,6}\right), 125$ (Cth, $\mathrm{C}_{2,5}$ ), 122 (Cpy, $\mathrm{C}_{3,5}$ ), $120\left(\mathrm{C}_{\mathrm{py}}, \mathrm{C}_{3}\right), 105\left(\mathrm{C}_{\mathrm{pyr}}, \mathrm{C}_{4}\right), 60\left(\mathrm{OCH}_{2}\right), 14$ $\left(\mathrm{C}, \mathrm{ArCH}_{3}\right)$ and $13\left(\mathrm{C}, \mathrm{CH}_{3}\right)$. Mass at $\mathrm{m} / \mathrm{z}=404$ $\left(\mathrm{M}^{+}, 90 \%\right)$ and $57\left(\mathrm{M}^{+}-347,100 \%\right)$.

Synthesis of 8-ethoxycarbonyl-9-methyl-5oxo-7-phenylpyrazolo[3",4"-c]pyrido [3',2':4,5]thieno[2,3-e]pyridine (16).

A mixture of compound $15(2 \mathrm{~g}, 5 \mathrm{mmol})$ in 1,4-dioxane $(20 \mathrm{ml})$, triethylamine $(1 \mathrm{ml})$ was added. The reaction mixture was heated under reflux for $5 \mathrm{~h}$. and then left to cool. The precipitate that formed was collected and recrystallized from dioxane to give brown crystals of compound 16. Yield: $65 \%$; m.p: $322{ }^{\circ} \mathrm{C}$. Anal. for $\mathrm{C}_{21} \mathrm{H}_{14} \mathrm{~N}_{4} \mathrm{O}_{3} \mathrm{~S}$ (402.43) Calcd.: C, 62.68; H, 3.51; N, 13.92 \%.Found: C, $62.55 ; \mathrm{H}, 3.42 ; \mathrm{N}, 13.80 \%$. IR $\left(\mathrm{cm}^{-1}\right)$ at 1716 for $(\mathrm{C}=\mathrm{O}$ groups $)$ with the disappearance absorption bands of $(\mathrm{NH})$. Mass at $\mathrm{m} / \mathrm{z}=402$ $\left(\mathrm{M}^{+}, 20 \%\right)$ and $363\left(\mathrm{M}^{+}-39,100 \%\right)$.

Synthesis of 3-banzoyl-8-ethoxycarbonyl-9methyl-5-oxo-7-phenylpyrazolo[3",4"-c] pyrido[3',2':4,5]thieno[2,3-e]pyridine (17).

To a suspension of compound 15 ( $2 \mathrm{~g}, 5$ $\mathrm{mmol})$ in 1,4-dioxane $(25 \mathrm{ml})$, piperidine (1 $\mathrm{ml})$ and benzaldehyde $(0.2 \mathrm{ml}, 2 \mathrm{mmol})$ was heated under reflux for $5 \mathrm{~h}$. The solid that formed on cooling was collected and recrystallized from dioxane to give pale yellow crystals of compound 17 . Yield: $76 \%$; m.p: $318{ }^{\circ} \mathrm{C}$. Anal. Calcd. for $\mathrm{C}_{28} \mathrm{H}_{20} \mathrm{~N}_{4} \mathrm{O}_{3} \mathrm{~S}$ (492.55): C, 68.28; H, 4.09; N, $11.37 \%$. Found: C, 68.10; H, 3.87; N, $11.21 \%$. IR (cm $\left.{ }^{1}\right)$ at 1710 for $(\mathrm{C}=\mathrm{O}$ groups $)$. Mass at $\mathrm{m} / \mathrm{z}=$ $493\left(\mathrm{M}^{+}, 70 \%\right)$ and $467\left(\mathrm{M}^{+}-25,100 \%\right)$.

Synthesis of 3-amino-11-ethoxycarbonyl-12methyl-5,8,-dioxo-10-phenylpyrralo [1"',2"'b]pyrazolo[3",4"-c]pyrido[3',2':4,5]

thieno[2,3-e]pyridine (18).

To a mixture 338 (2 g, $5 \mathrm{mmol})$, ethyl cyanoacetate $(1 \mathrm{ml}, 10 \mathrm{mmol})$ in 1,4-dioxane $(20 \mathrm{ml})$ and triethylamine $(1 \mathrm{ml})$ was added. The reaction mixture was heated under reflux for $5 \mathrm{~h}$. and then left to cool. The solid that formed was collected and recrystallized from ethanol-dimethylformamide $(1: 1)$ mixture to give pale yellow crystals of compound 18 . Yield: $85 \%$; m.p: $338{ }^{\circ} \mathrm{C}$. Anal. Calcd. for 
$\mathrm{C}_{24} \mathrm{H}_{17} \mathrm{~N}_{5} \mathrm{O}_{4} \mathrm{~S}$ (471.49): C, 61.14; H, 3.63; N, 14.85 \%. Found: C, 60.89; H, 3.49; N, 14.64 $\%$. IR $\left(\mathrm{cm}^{-1}\right)$ at 3420,3350 for $\left(\mathrm{NH}_{2}\right)$, at 1720 for $\left(\mathrm{C}=\mathrm{O}\right.$ groups). ${ }^{1} \mathrm{H} \mathrm{NMR} \mathrm{CDCI}_{3}$ at $\delta=7.9$ (s, H, NH pyridine), 7.1-7.7 (m, 7H, aromatic and $\mathrm{NH} 2$ protons), 5.1 (s, $\mathrm{H}, \mathrm{CH}$ pyrralo), 4.0 (q, $\left.2 \mathrm{H}, \mathrm{OCH}_{2}\right), 2.8\left(\mathrm{~s}, 3 \mathrm{H}, \mathrm{CH}_{3}\right.$ at $\left.\mathrm{C} 10\right)$ and 1.3 (t, $3 \mathrm{H}, \mathrm{CH}_{3}$ ester). $13 \mathrm{C} \mathrm{NMR} \mathrm{CDCI}_{3}$ at $\delta=187$ $\left(\mathrm{C}_{\mathrm{pyrr}}, \mathrm{C}=\mathrm{O}\right), 165\left(\mathrm{C}_{\mathrm{py}}, \mathrm{C}_{3}\right), 167\left(\mathrm{C}_{\mathrm{ester}}, \mathrm{C}=\mathrm{O}\right)$, $162\left(\mathrm{C}_{\mathrm{py}}, \mathrm{C}_{6}\right), 157\left(\mathrm{C}_{\mathrm{pyrr}}, \mathrm{C}_{3}\right), 158\left(\mathrm{C}_{\mathrm{pyr}}, \mathrm{C}_{6}\right)$, $138\left(\mathrm{C}_{\mathrm{ph}}, \mathrm{C} 1\right), 129\left(\mathrm{C}_{\mathrm{ph}}, \mathrm{C}_{2,3,4,5,6}\right), 126\left(\mathrm{C}_{\mathrm{py}}\right.$, $\left.\mathrm{C}_{5,6}\right), 125\left(\mathrm{C}_{\mathrm{th}}, \mathrm{C}_{3}\right), 122\left(\mathrm{C}_{\mathrm{py}}, \mathrm{C}_{2,3}\right), 105\left(\mathrm{C}_{\mathrm{pyr}}\right.$, $\left.\mathrm{C}_{4}\right), \quad 84\left(\mathrm{C}_{\mathrm{pyrr}}, \mathrm{C}_{4}\right), 60\left(\mathrm{C}, \mathrm{OCH}_{2}\right), 14(\mathrm{C}$, $\left.\mathrm{ArCH}_{3}\right)$ and $13\left(\mathrm{C}, \mathrm{CH}_{3}\right)$. Mass at $\mathrm{m} / \mathrm{z}=472$ $\left(\mathrm{M}^{+}+1,60 \%\right)$ and $296\left(\mathrm{M}^{+}-175,100 \%\right)$.

Synthesis of 8-ethoxycarbonyl-9-methyl-5oxo-7-phenyl-3-N-(phenylthiomethylamido) pyrazolo[3",4"-c]pyrido[3',2':4,5]thieno [2,3elpyridine (19).

A mixture of compound 15 ( $2 \mathrm{~g}, 5 \mathrm{mmol})$, phenylisothiocyanate $(0.67 \mathrm{ml}, 5 \mathrm{mmol})$ in $1,4-$ dioxane $(20 \mathrm{ml})$ and triethylamine $(1 \mathrm{ml})$ was heated under reflux for $5 \mathrm{~h}$, and then left to cool. The precipitate that formed was collected and recrystallized from dimethylformamide to give pale yellow crystals. Yield: $60 \%$; m.p: $130{ }^{\circ} \mathrm{C}$. Anal. for $\mathrm{C}_{28} \mathrm{H}_{19} \mathrm{~N}_{5} \mathrm{O}_{3} \mathrm{~S}_{2}$ (537.61) Calcd.: C, 62.55; H, 3.56 ; N, 13.02 \%. Found: C, 62.21; H, $3.38 ; \mathrm{N}, 13.09 \%$. IR $\left(\mathrm{cm}^{-1}\right)$ at 3280 for $(\mathrm{NH})$, at 1720 for $(\mathrm{C}=\mathrm{O}$ groups $)$. Mass at $\mathrm{m} / \mathrm{z}=462\left(\mathrm{M}^{+}-75,80 \%\right)$ and 438 $\left(\mathrm{M}^{+}-99,100 \%\right)$.

Synthesis of 9-ethoxycarbonyl-4-hydrothio 10-methyl-2,5,6-trioxo-8-phenylindolazino [3",4"-c]pyrido[3',2':4,5]thieno[2,3-e] pyridine (20).

A mixture of compound $15(2 \mathrm{~g}, 5 \mathrm{mmol})$, ethyl cyanoacetate $(1.1 \mathrm{ml}, 10 \mathrm{mmol})$ in $1,4-$ dioxane $(20 \mathrm{ml})$ with elemental sulfur $(3.2 \mathrm{~g}$, $10 \mathrm{mmol})$ and triethylamine $(1 \mathrm{ml})$ was added. The reaction mixture was heated under reflux for $5 \mathrm{~h}$. and then left to cool. The precipitate that formed was collected and recrystallized from dioxane to give brown crystals. Yield: 60 $\%$; m.p: $300{ }^{\circ} \mathrm{C}$. Anal. Calcd. for $\mathrm{C}_{25} \mathrm{H}_{16} \mathrm{~N}_{4} \mathrm{O}_{5} \mathrm{~S}_{3}$ (548.61): C, 54.73; H, 2.94; N, $10.21 \%$. Found: C, 54.65; H, 2.64; N, $10.05 \%$. IR $\left(\mathrm{cm}^{-1}\right)$ at 1710 for $\left(\mathrm{C}=\mathrm{O}\right.$ groups). ${ }^{1} \mathrm{H}$ NMR $\left(\mathrm{CDCI}_{3}\right)$ at $\delta=7.2-7.4(\mathrm{~m}, 6 \mathrm{H}$, aromatic and $\mathrm{CH}$ at $\mathrm{C}_{4}$ protons), 4.0 (q, $\left.2 \mathrm{H}, \mathrm{OCH}_{2}\right), 2.5$ (s, $3 \mathrm{H}, \mathrm{CH}_{3}$ at $\left.\mathrm{C}_{13}\right), 1.5(\mathrm{~s}, 2 \mathrm{H}, 2 \mathrm{SH})$ and $1.0(\mathrm{t}$, $3 \mathrm{H}, \mathrm{CH}_{3}$ of ester). Mass at $\mathrm{m} / \mathrm{z}=550\left(\mathrm{M}^{+}+2\right.$, $90 \%)$ and $485\left(\mathrm{M}^{+}-63,100 \%\right)$.

Synthesis of 8-ethoxycarbonyl-9-methyl-5oxo-7-phenyl-3-(N-thiophenylthioamide) pyrazolo[3",4"-c]pyrido[3',2':4,5]thieno [2,3elpyridine (21).

To mixture of compound 15 ( $2 \mathrm{~g}, 5 \mathrm{mmol})$, phenylisothiocyanate $(0.67 \mathrm{ml}, 5 \mathrm{mmol})$ in 1,4 dioxane $(20 \mathrm{ml})$ with elemental sulfur (3.2 gm., $10 \mathrm{mmol})$ and triethylamine $(1 \mathrm{ml})$ was added. The reaction mixture was heated under reflux for $5 \mathrm{~h}$. and then left to cool. The precipitate that formed was collected and recrystallized from dioxane to give dark brown crystals of compound 21. Yield: $60 \%$; m.p: $120^{\circ} \mathrm{C}$. Anal. Calcd. for $\mathrm{C}_{28} \mathrm{H}_{21} \mathrm{~N}_{5} \mathrm{O}_{3} \mathrm{~S}_{3}$ (571.69): C, 58.83; H, 3.70; N, 12.25 \%.Found: C, 58.58; H, 3.57; $\mathrm{N}, 12.01 \%$. IR $\left(\mathrm{cm}^{-1}\right)$ at 1710 for $(\mathrm{C}=\mathrm{O}$ groups). ${ }^{1} \mathrm{H} \mathrm{NMR}\left(\mathrm{CDCI}_{3}\right)$ showed at $\delta=7.2$ 7.4 (m, 10H, aromatic protons), 4.3 (t, H, CHS ), 4.1 (q, $\left.2 \mathrm{H}, \mathrm{OCH}_{2}\right), 2.4\left(\mathrm{~s}, 3 \mathrm{H}, \mathrm{CH}_{3}\right.$ at $\left.\mathrm{C}_{9}\right)$ and $1.5(\mathrm{~s}, 2 \mathrm{H}, 2 \mathrm{SH})$ and $0.9\left(\mathrm{t}, 3 \mathrm{H}, \mathrm{CH}_{3}\right.$ of ester). Mass at $\mathrm{m} / \mathrm{z}=571(\mathrm{M}+, 10 \%)$ and $256\left(\mathrm{M}^{+}\right.$. $315,100 \%)$. 
Synthesis of 8-ethoxycarbonyl-9-methyl-5oxo-7-phenyl-3-phenyldiazinopyrazolo [3",4"-c]pyrido[3',2':4,5]thieno[2,3-e] pyridine (22).

To a cold solution $\left(0-5^{\circ} \mathrm{c}\right)$ of compound 15 $(2 \mathrm{~g}, 5 \mathrm{mmol})$ in ethanol $(20 \mathrm{ml})$ containing sodium hydroxide $(1 \mathrm{~g})$ and diazotized aniline was gradually added while stirring during 5 min. The formed upon cooling in ice-bath was collected and recrystallized from 1,4-dioxane to give reddish brown crystals of compound 22. Yield: $76 \%$; m.p: $140{ }^{\circ} \mathrm{C}$. Anal. for $\mathrm{C}_{27} \mathrm{H}_{18} \mathrm{~N}_{6} \mathrm{O}_{3} \mathrm{~S}$ (506.54) Calcd: C, 64.02; $\mathrm{H}$, 3.58 ; N, 16.59 \%.Found: C, 64.15; H, 3.36; N,
$16.31 \%$. IR $\left(\mathrm{cm}^{-1}\right)$ at 1710 for ( $\mathrm{C}=\mathrm{O}$ groups). ${ }^{1} \mathrm{H}$ NMR $\left(\mathrm{CDCI}_{3}\right)$ at $\delta=6.8-8.0(\mathrm{~m}, 10 \mathrm{H}$, aromatic protons), $4.0\left(\mathrm{q}, 2 \mathrm{H}, \mathrm{OCH}_{2}\right), 2.6(\mathrm{~s}$, $3 \mathrm{H}, \mathrm{CH}_{3}$ at $\mathrm{C}_{8}$ ) and 1.0 (t, $3 \mathrm{H}, \mathrm{CH}_{3}$ of ester). Mass at $\mathrm{m} / \mathrm{z}=507\left(\mathrm{M}^{+}+1,20 \%\right)$ and $77\left(\mathrm{M}^{+}\right.$. $429,100 \%)$.

\section{3- Results}

\section{Biological activities:}

Study all new compound derivative were screened in vitro for antimicrobial activity against three species of bacteria (E.colie, P.seudemones and S.aurous) and three fungal species of (Aspergillus, Pencillium and Clado) using wells method. (Table 1).

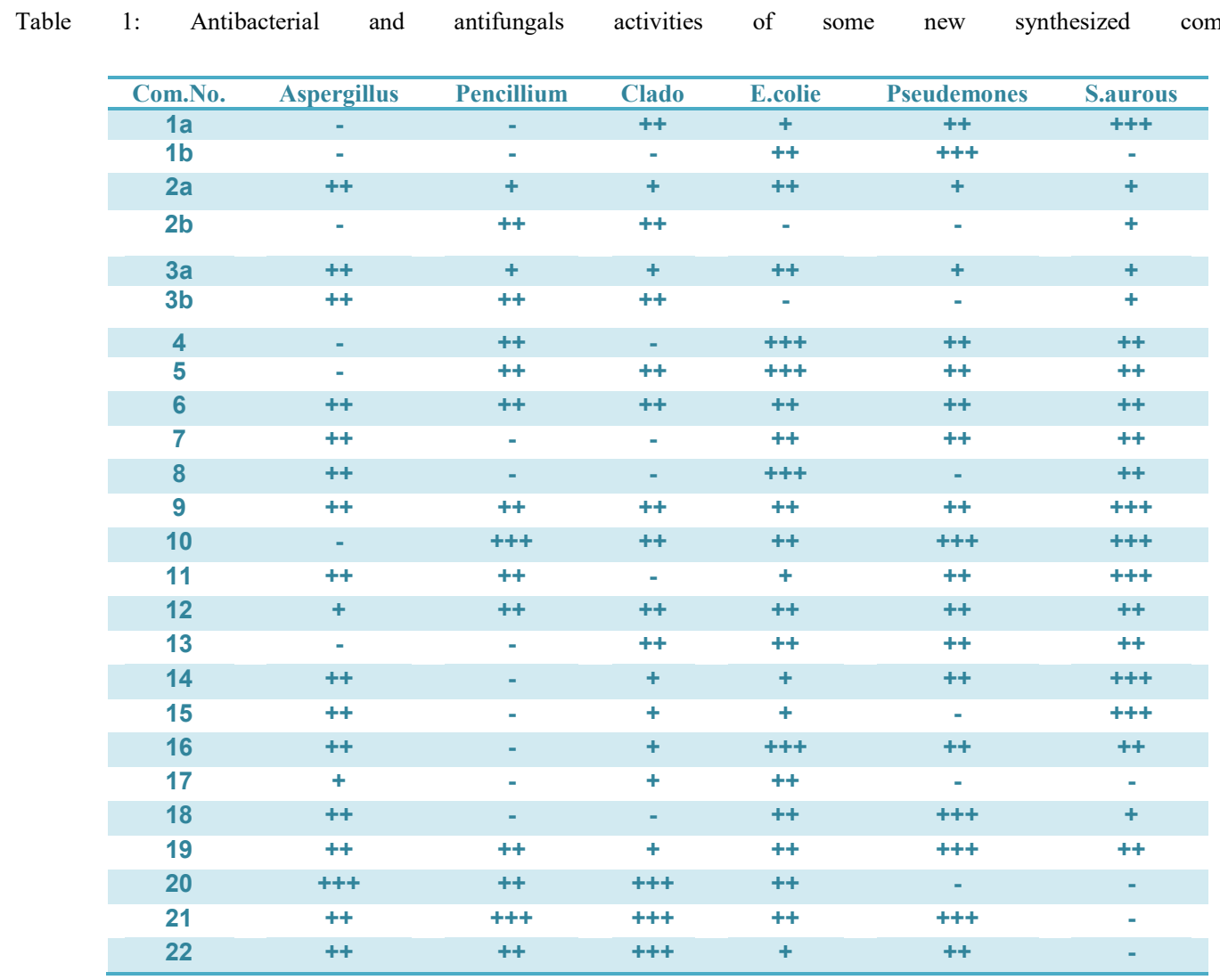

-: No activity; +: moderate activity (inhibition zone 5-10 mm); ++: strong activity (inhibition zone 11-15 mm); +++: very strong activity (inhibition zone 16-20 mm). 


\section{4-REFERENCES:}

[1] Rushit K., Manish M., Chetana R. and Jatin

J. U., "Synthesis, characterization and antimicrobial activity of substituted tetrahydrothieno $\quad[2,3-\quad$ c]pyridin-2-yl)urea derivatives." Journal of Chemical and Pharmaceutical Research, 4(3),

1566-1572, (2012).

[2] E. A. Bakhite, A. E. Abdel-Rahman, O. S.Mohamed, and E. A. Thabet.,"Synthesis and Reactions of New Thienopyridines, Pyridothienopyrimidines and Pyridothieno triazines.",Bull. $\quad$ Korean Chem. Soc., 23, 12. $1709,(2002)$

[3] Eman R. K, M. A. El-Hashash, Mowafea A. S., Hemat S. K., and Naiera A. M. A. W., " Synthesis and Reactions of Some Novel Nicotinonitrile Derivatives for Anticancer and Antimicrobial Evaluation." Acta Chim. Slov., 56, 908-919, (2009).

[4] Nora M. R.," Synthesis and reactions of 4trifluoromethyl-3-cyanopyridine-2(1H)thione/one derivatives." Journal of Sulfur Chemistry, 32, 6, 611-622, (2011)

[5] A. Y. Hassan1, M. T. Sarg, M. M. Said and S. A. El-Sebaey., " Utility of thieno[2,3b]Pyridine derivatives in the synthesis of some condensed heterocyclic compounds with expected biological activity." Universal Organic Chemistry, 1-15 (2013).
[6] Nora M. R, S., H. A. and Hussein. F. Z.,"Synthesis and Antimicrobial Evaluation of Some New Heterocyclic Compounds from Thienopyridine and Pyrazolopyridine Derivatives." International Journal of Advanced Research 2, 1, 446-455, (2014).

[7] Ali K. El-L., Shadia M. A. and Emtithal A. El- S., " Synthesis of Some Aryl Thienopyridine, Pyridothienopyrimidine, and Pyridothieno triazolopyrimidine Derivatives." Life Science Journal; 9(1), (2012).

[8] Ahmed M. M. El-Saghiera, Fathy S. M., Mahmoud F. F., Nabil A. S., Khaled M. Kr eddanc, Somaia O. El-Tierb , Hind Bin Hussienc., " Synthesis and Biological Evaluation of Some New Thienopyridine and Thienopyrimidine Derivatives." Jordan Journalof Chemistry, 3 3, 223-232, ( 2008).

[9] Naglaa A. A-H, Ashraf, M. M., Abd El-Galil E. A., Mohamed M. A., " Antiarrhythmic Activities of Some Newly Synthesized Tricyclic and Tetracyclic Thienopyridine Derivatives.",Sci Pharm.; 77; 539-553 (2009)

[10] Ali. K. K. El-louh., " O- Aminonitrile in Heterocyclic Chemistry Synthesis of Thienopyridine, pyridothieno pyrimidine, pyridothieno[1,2,4]triazolopyrimidine,pyridoth ienopyridine Derivatives.", Natural Sciences, 10, 1-20, ( 2008).

[11] Azza M. Abdel-Fattah, Fawzy A. Attaby and Labeeb M. Shaif ., " Synthesisof Some Fused Heterocyclic Compounds Based on 1-(1- 
Benzofuran-2-yl)-3-(furan-2-yl)prop-2-en-1one." International Journal of Scientific \& Engineering Research, 4, 11, 1831 (2013)

[12] Karam A. El-S. Rehab A. I., "new approache for the synthesis and antitumor evaluation of pyridine, thieno[3,4-c] pyridine, pyrazolo $[3,4-$ $b]$ pyridine and pyrido[3,4- $d]$ pyridazine derivatives.", Eur. Chem. Bull., , 2(8), 530-537, (2013)

[13] Rafat M. M. , and Fatma O. Al-farouk., Anti-Tumor and Anti-Leishmanial Evaluations of Novel Thiophene Derivatives Derived from the Reaction of Cyclopentanone with Elemental Sulphur and Cyano-Methylene Reagents." Organic Chem, 1-6 (2012)

[14] Mahmoud A. M., " Synthesis of Some New Bipyridines, Thieno[2,3-b]pyridines, and
Pyrazolo[3,4-b]pyridines., "49 , 200-202, (2012).

[15] Mohamed A.M. Gad-Elkareema, Azza M. Abdel-Fattahb and Mohamed A.A. Elneairy Pyridine-2(1H)-thione in heterocyclic synthesis: synthesis and antimicrobial activity of some new thio-substituted ethyl nicotinate, thieno[2,3-b]pyridine and pyridothieno pyrimidine derivatives." Journal of Sulfur Chemistry, 32, 3, 273-286, ( 2011).

[16 ] Shrikant B. Kanawade, Shivaraj P. P, Prashant S. Nikam, Sachin A. Gangurde,Madhukar N. Jachak, and Raghunath B. Toche "Synthesis of New Thieno[2,3-d]pyrimidines, Thieno[3,2e]pyridines, and Thieno[2,3-d][1,3]oxazines.", Journal of Heterocyclic Chemistry,49, 363-369 (2012). 


\section{تخليق وتقدير الفعالية البيولوجية لبعض المركبات الجديدة غير متجانسة الحلقة المتعلقة بمشتقات الثينوبيريدين و الثينوبير ميدينين.

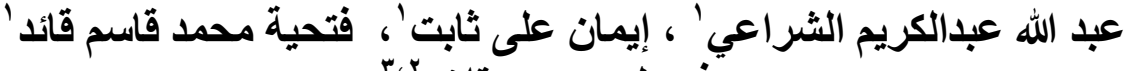

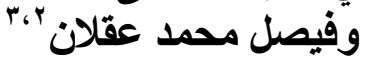

fethiyemohammed@gmail.com

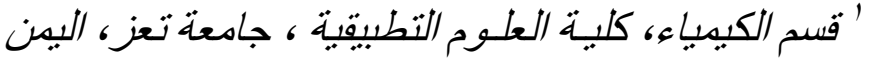

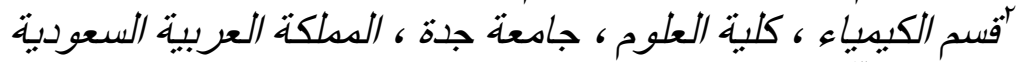

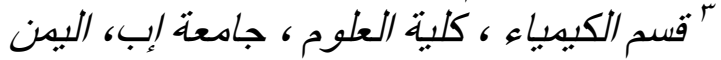

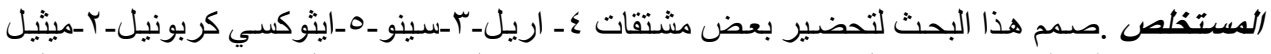

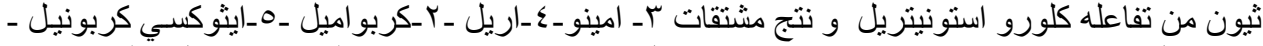

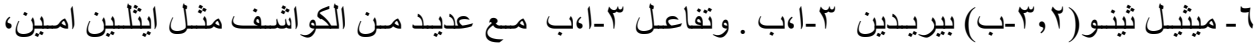

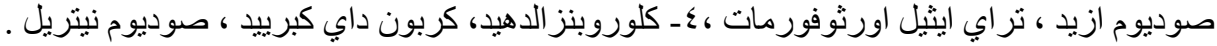

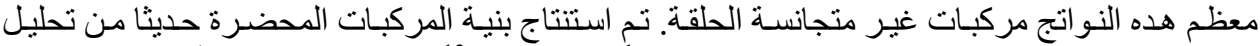

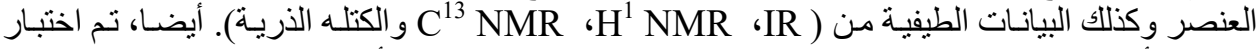
در اسة الأنشطة البيولوجية لجميع المركبات التي تم توليفها في المختبر لأنشطة مضـادة للميكروبـات مثنل

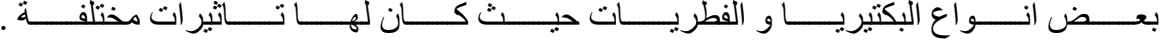

